\title{
EDITORIAL \\ Las preocupaciones de la Historia
}

\author{
Francisco Alberto Pérez Piñón"
}

Director

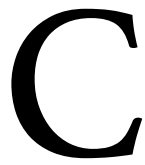

on este título, a manera de provocación, abordamos la editorial del presente número de Debates por la Historia, correspondiente al periodo julio-diciembre de 202I. Se destaca, en un primer momento, la aclaración sobre este título; enseguida la narrativa del Seminario Internacional de Teoría de la Historia, coordinado por los doctores Daniel Ovalle Pasten y Fernando Betancourt, al cual tuvimos la fortuna de ser invitados como Cuerpo Académico de Historia e Historiografía de la Educación; y, finalmente, como es costumbre, una breve presentación de los artículos que se publican en el presente número.

El título que encabeza la presente editorial intenta responder a los cuestionamientos y diatribas que se discuten en la actualidad en el campo de la historia e historiografía, esta última considerada como el concepto ligado a la descripción que se hacen de los acontecimientos y a la forma en cómo se presentan en la escritura, para darse a conocer a los interesados en este campo.

Profundizando en el título, debemos externar que la Historia, entendida como acontecimientos en el tiempo, no tiene preocupaciones de ningún tipo, ya que esos acontecimientos no cambian, están allí como ese pasado que será descubierto por quienes se preocupen por él, ya sea quienes indagan en un pasado histórico o un pasado práctico, y es aquí en donde surge la

\footnotetext{
Universidad Autónoma de Chihuahua. Correo electrónico: aperezp@uach.mx
} 
preocupación por conocer ese pasado, pero son los investigadores de este campo -los historiadores- quienes van a revivir esos acontecimientos. El pasado es removido y son ellos quienes deben estar preocupados por dar a conocer esa descripción, por la comprensión y por el sentido de rescatar acontecimientos del pasado.

Se mencionó anteriormente el pasado práctico e histórico. El primero tiene relación con el presente y proyección a ese horizonte de espera, a la manera como Koselleck lo expresa con sus categorías de espacio de experiencia y horizonte futuro (Blanco, 2012). En sí, serán los historiadores y demás investigadores quienes se ocupen de direccionar ese pasado, de rescatar lo útil y práctico que sirva a esta sociedad del siglo XXI. Por otro lado, el rescate del pasado histórico vendría a ser todo nuestro pasado, habrá que buscarle el sentido de su recuperación y narración en el presente, qué sentido se le asigna más allá de lo que se ha mencionado durante tanto tiempo, de que el pasado -nuestro pasado- tiene que ver con la identidad. Sin embargo, debemos decirlo, existen demasiados productos de investigación que redimen el pasado y que como antigüedades los tenemos presentes en estantes, librerías y demás aparadores, pero la mayoría de las veces reflejan un pasado muerto, sin relación con el presente y mucho menos nos sirven para orientarlo y direccionarlo.

Es tiempo que los interesados en el campo de la Historia se aboquen a reconstruir ese pasado -sea el histórico o el práctico- en busca de la resolución de problemas de la sociedad actual; de lo contrario seguiremos en lo mismo, en la acumulación cognitiva del pasado para transformarlo en contenidos programáticos, que serán 8 trabajados en las aulas por profesores y estudiantes de forma los involucrados en este terreno.

Sin duda, la preocupación mayúscula de la historia y los historiadores debe ser formativa, en donde se realicen las articulaciones de pasado, presente y futuro; en busca de lo reflexivo 
y pragmático; de la resolución de problemas que aquejan a las sociedades; de justicia, igualdad y equidad de géneros; de la distribución de los recursos económicos necesarios para la reproducción de la vida material y espiritual; en sí, en busca de ese horizonte Koselleckiano de la construcción de un mejor futuro, porque la historia como disciplina debe de abonar desde este presente, considerando el pasado.

A manera de cierre con lo nominal del título, se externa que la revista Debates por la Historia trabaja en busca de categorías y discusiones actuales en este campo y su utilización en los objetos de discusión en el área de la educación, lo que ha venido a establecerse como norma, que forma parte de la forja del punto de vista de esta revista científica.

\section{El Seminario Internacional de Teoría de la Historia}

Como anteriormente se mencionó, recibimos la invitación para el Cuerpo Académico de Historia e Historiografía de la Educación responsable de la edición de esta revista- para participar en el Seminario Internacional de Teoría de la Historia que, de entrada, tenemos que mencionar que es un espacio en el que los participantes están marcando las tendencias actuales en el campo de la investigación histórica, principalmente en la parte teórica. A ellos, con atrevimiento, también se les ubica como posmodernistas, precisamente por el rompimiento que tienen con las viejas y anacrónicas prácticas de investigación; así como por no abocarse al pasado en busca de objetividades ni verdades permanentes. Este grupo de académicos realiza un esfuerzo intelectual de posicionamiento de la Historia en esas interrelaciones temporales. Así se les visualiza y esperamos demostrarlo con la presente narrativa; pero, ¿quiénes son los coordinadores del seminario? 
Al Dr. Daniel Ovalle Pastén se le conoce como un interesado en posicionar -desde la teoría- a la historia del tiempo presente, según la tesis presentada en 2019 para obtener el grado de doctor en la Universidad de Chile, denominada La escritura de la memoria como régimen historiográfico. El historiador "afectado por el pasado". En este documento ofrece un posicionamiento epistémico robusto con las citas a Francois Hartog, en los regímenes de historicidad que privilegian el estudio de los acontecimientos del tiempo presente; Paul Ricoeur, quien recupera el establecimiento de ese pacto de verdad entre quien escribe y quien lee los productos históricos, la narrativa y la figuración de los acontecimientos; Michel de Certeau, que se apoya en la producción de la escritura de la historia desde el lugar de su fabricación; Maurice Halbwachs, con el posicionamiento de la memoria y la historia para ubicar el régimen de historicidad del presentismo; entre otros.

La postura del Dr. Ovalle sin duda está direccionada hacia la interdisciplinariedad de la historia, en donde las fronteras disciplinares se difuminan y tienden a sustituirse por visiones más holísticas, donde los préstamos metódicos y teóricos están presentes como herramientas y se apoyan, para profundizar en el objeto de estudio. Innegablemente él es un estudioso y apasionado de la teoría de la historia y está aportando a las discusiones actuales de la disciplina.

Fernando Jesús Betancourt Martínez es Doctor en Historia y Etnohistoria por la Escuela Nacional de Antropología e Historia. Actualmente tiene como adscripción principal el Instituto de Investigaciones Históricas de la Universidad Nacional Autónoma de México (UNAM) y desarrolla las siguientes líneas de investigación: Complejidad social, teoría de sistemas y cognición histórica; Psicoanálisis freudiano: la autorreferencia social en perspectiva histórica y Epistemología e historia.

En su desempeño como coordinador del Seminario, el Dr. Betancourt no deja de llamar la atención con sus acertadas 
preguntas en busca de nuevos paradigmas y por la crítica al agotamiento de las prácticas de los escritos historiográficos carentes de problematizaciones y, por lo tanto, de validez en la actualidad. Participa en la búsqueda de categorías que en este tiempo se discuten en el campo de la historia e historiografia, haciendo un comparativo entre los siglos pasados y el actual; sin dejar de lado el giro pragmático que expresa en uno de sus múltiples escritos:

Para ello introduce el denominado enfoque pragmático que, desde la filosofía de la ciencia contemporánea, se convierte en elemento que tiende a sustituir la anterior discusión epistemológica. Resalta con este enfoque el carácter comunicativo propio de la racionalidad operante en la base disciplinaria y la exigencia de contextualización que se desprende de él. Una forma de enfrentar dicha exigencia se localiza en el concepto de regímenes de historicidad (Betancourt, 2006, p. I03).

Con la cita anterior, el Dr. Betancourt deja en claro el valor asignado a las intersubjetividades de los investigadores, quienes deben acercarse más al presente. Plantea la crítica a la racionalidad de las sociedades actuales, sin abandono de la reflexividad y pragmatismo de la disciplina. Hace referencia a Françoise Hartog en su citado texto de los regímenes de historicidad: presentismo y experiencias en el tiempo. Nos induce a la necesidad de estudiar el presente, este largo y único presente.

Sin duda, los cuestionamientos que el Dr. Betancourt realiza en las sesiones de trabajo del Seminario son de gran valor, ya que despiertan la curiosidad y el deseo de abundar en las teorías de la historia, para buscar su aplicación en trabajos que parten de los acontecimientos y -sobre todo- de aquellos que provocan la discusión y reflexiones por su relación con ese pasado-presente y futuro. 


\section{Dinámica del seminario}

El Seminario Internacional es conducido por los coordinadores ya mencionados, quienes se dan a la tarea de invitar a personalidades destacadas que pertenecen a disciplinas como la antropología, filosofía, literatura, teoría de la historia, entre otras; pero que tienen como objeto focal el estudio de la teoría de la historia.

Al inicio de cada sesión de trabajo se hace una presentación de quien realizará la exposición. Previamente el invitado o invitada envía un documento de lectura relacionado con el tema que se tratará en la sesión. Se realiza una exposición de aproximadamente sesenta minutos y posteriormente un bloque de preguntas y respuestas, que regularmente duran otros sesenta minutos, ya que la actividad está programada para ciento veinte minutos. La exposición del ponente y las preguntas del público son de lo más innovadoras y útiles para los interesados en este campo, además de tener la oportunidad de conocer -en la virtualidad- a los personajes que alguna vez hemos leído. Las sesiones del seminario se programan una vez al mes y los participantes son de distintas nacionalidades (Chile, España, Francia, Argentina, México, Alemania, entre otros).

La escucha de ponentes y participantes permite conocer las visiones, problemáticas, intereses y abordajes de lo que están trabajando en distintos países, situación que ayuda a conocer nuevas visiones para el crecimiento y profundidad en el campo de la historia e historiografía.

\section{Reseña de las temáticas y ponentes}

Ante lo estrecho de la escritura de la presente editorial, solo se narra de manera breve, las intervenciones de los ponentes y el tema que presentaron durante el lapso de tiempo en que hemos tenido 
oportunidad de participar. Javier Fernández Sebastián es catedrático de Historia del Pensamiento Político en la Universidad del País Vasco (Bilbao). Fundador del Grupo de Historia intelectual de la política moderna de la UPV/EHU (GIU I8/2I5) y de la red Iberconceptos. Dictó la conferencia denominada "Los silencios de la historia", título que se enmarca dentro de las temáticas que trata su reciente libro Historia conceptual en el Atlántico ibérico. Lenguajes, tiempos, revoluciones (FCE, 202I). Como el nombre del texto lo indica, y como él lo mencionó en su conferencia, es un seguidor fiel de Reinhart Koselleck, porque discute que, al no tomar en cuenta que los conceptos también tienen historia, se descontextualizan en las narrativas. Menciona que las continuidades y las rupturas enmarcadas en los lenguajes en el tiempo y las revoluciones, proporcionan una brecha infranqueable entre los acontecimientos $y$ los conceptos. Cuestiones interesantes fueron debatidas en esta sesión del Seminario que -sin duda- abonan a la realización teórica y metodológica de trabajos de actualidad en el campo de la historia.

El Dr. Guillermo Zermeño, del Colegio de México (COLMEX), y la Dra. Ingrid Simson de la Freie Universität, Berlín, presentaron el libro del que son editores: La Historiografía en tiempos globales (2020) de la Editorial Tranvía. Guillermo Zermeño, según lo publicado en la página del COLMEX, es Doctor en Ciencias Sociales con especialidad en Historia por la Universidad Johann Wolfgang Goethe, Alemania (1983). Desarrolla investigaciones en el campo de la historia cultural y conceptual, la historiografía y la teoría de la historia, con especial énfasis en la historia moderna de México. Fue director de la revista científica Historia y Grafía, desde su fundación hasta el número nueve $\mathrm{y}$, a partir del número Io, asumió como director el Dr. Alfonso Mendiola Mejía, quien es uno de los participantes en este Seminario y en sus participaciones ha hecho alusión a su gran apego en la investigación al historiador francés Michel de Certeau.

Al no encontrar más información relacionada con la Dra. Simson, pasamos a la reseña del texto que abordaron en la sesión de trabajo 
y para ello rescatamos la gran relación e interconexión que existe entre los distintos mundos y culturas, lo que explica que cuando ocurre algún cambio, impacta en las demás regiones. En la actualidad las reflexiones que realizan los autores del texto derivan en el protagonismo de la globalización. El capitalismo global se manifiesta en la multiplicidad de discursos que en la actualidad se producen en el campo de la historia e historiografía.

La Dra. María Elisa Fernández Navarro es profesora de la Universidad de Chile, de quién se menciona brevemente su formación académica. De 1997-I998 cursó el Post doctorado en el Department of History and Center of Latin American Studies de la University of Michigan, Estados Unidos. En los años de 1990-1996 obtuvo el Ph. D. en Historia con la tesis titulada Beyond Partisan Politics in Chile: The Carlos Ibañez Period and the Politics of Ultra Nationalism during 1952-1958 en la University of Miami, Florida, bajo la dirección del Dr. Steve Stein. En los años de 1984-1989 cursó estudios de Licenciatura en Historia en la Pontificia Universidad Católica de Chile, en la especialidad de Historia Política de Latinoamérica, donde fue graduada con distinción (Universidad de Chile, s/f). Participó en la sesión del Seminario Internacional con la conferencia titulada "La evolución de la propuesta de Joan Scott sobre género y su aporte a los discursos teóricos que hoy nos ocupan."

La intervención de la Dra. Fernández fue brillante y más que una discusión acabada sobre los estudios de género -por lo mucho que se ha venido tratando el tema hasta hoy- dejó canales abiertos para la discusión y las reflexiones. Señaló la urgencia de analizar el 14 concepto y situarlo más allá de los límites de la subordinación al poder, la igualdad y la equidad; girando hacia una interpretación más dinámica, que haga alusión a las luchas y expectativas de los distintos géneros en este mundo posmodernista, donde es necesario situar el concepto ante las grandes diferencias que se presentas al interior de las regiones, países y el mundo. 
La Dra. María Norma Durán Rodríguez Arana es profesora de la Universidad Autónoma Metropolitana y cultiva las líneas de investigación Historiografía del siglo XVI; Historia de la evangelización, siglos XVI y XVII, e Historia de la religiosidad medieval y moderna. De su producción investigativa destacamos los libros en los que ha participado como coordinadora: Inventando a Hayden White. Imaginación y narrativas (2020), Estudios Culturales. Voces representaciones y discursos (20I7), Epistemología histórica e historiografía (2017) y Formas de hacer la historia. Historiografía grecolatina y medieval (20I6) (UAM-AZC, s/f).

La Dra. Verónica Tozzi Thompson es profesora de Filosofía de la Historia en la Universidad de Buenos Aires, Argentina, y Epistemología de las Ciencias Sociales en la Universidad Tres de Febrero. Algunas de sus publicaciones recientes son: La historia según la nueva filosofía de la historia (2009), Calculando la experiencia bélica de Malvinas. La heurística y la historia como una promesa incumplida (2009) y Pragmatist Contributions to a New Philosophy of History (20I2). Sus principales temas de interés son Filosofía de la historia y las ciencias sociales, Epistemología del testimonio y Política de la memoria (Academia, 202I).

La sesión del seminario -donde participaron las docetas María Norma Duran y Verónica Tozzi- estuvo referida a la presentación del libro Inventando a Hayden White. Imaginación y narrativas (202I). La obra nos lleva a retomar el acercamiento entre la literatura y la historia, al considerar esta última como una invención discursiva, idea con la que estigmatizaron a Hayden White. Este autor vino a dar frescura a la Historia actual en los años sesenta y en la actualidad sigue siendo discutido y reinterpretado. La invención de la historia se posicionó como parte de las narrativas que hacen los historiadores y como una forma de redactar los productos de investigación histórica. La narración es el medio por el cual pervive el acontecimiento, no como la realidad en sí misma, pues esta es convertida en discurso. 
La Dra. María Inés La Greca, de la Universidad Nacional Tres de Febrero y Universidad de Buenos Aires, Argentina, presentó en el Seminario Internacional la conferencia "La pregunta actual por el acontecimiento, el relato y la historización: una aproximación a la movilización feminista Argentina". Con respecto a su producción, hacemos aquí el listado de algunos trabajos a los que se puede tener acceso en línea: El ambiguo valor de la narratividad en Hayden White: cómo seguir escribiendo (intrasitivamente) la historia; Experiencia y estructura narrativa; El concepto de campo histórico como construcción lingüístico-ficcional-hipotética en Hayden White; Entre la ironía y el romance: pasado, presente y futuro de la historia narrativista; entre otros (Metahistorias, s/f).

Ante la imposibilidad de hacer la narrativa detallada de todo lo tratado por la Dra. María Inés en la sesión de trabajo, solo dejamos asentado en este escrito que ella es una seguidora de Hayden White y de sus tesis narrativistas de los acontecimientos, a la vez que defensora de los derechos de las mujeres en Argentina, definiéndose como feminista comprometida con esta sociedad del siglo XXI.

A manera de cierre, se manifiesta que el Seminario Internacional coordinado -como ya se dijo- por los doctores Daniel Ovalle y Fernando Betancourt, está siendo un hito para la difusión de la teoría de la historia, mostrando los avances y desarrollos más significativos de este siglo. La aportación más importante está en la difusión de la teoría de la historia que se está dando a conocer por los autores productores de estos enfoques teóricos vanguardistas.

Reseña de los artículos que se publican en este número

Pasando a la presentación de los artículos que se incluyen en el presente número de la revista, tenemos en primer término el trabajo titulado La enseñanza de la Historia y los libros de texto: producción, posibilidades y desafíos para la formación del profesorado, que nos 
traslada a la República de Brasil, lugar en donde se realiza la narrativa de cómo ha sido la producción de textos de educación básica y la forma en que son trabajados por los niños y los profesores; los desafíos, obstáculos y logros. La metodología utilizada en la investigación está basada en una exitosa búsqueda de información relacionada con la temática. La problematización en la aplicabilidad de los textos de historia en educación básica deriva en la necesidad de realizar otros planteamientos que conlleven a que profesores y estudiantes arriben a la reflexión y el encuentro del sentido de los contenidos cuando utilizan dichos materiales.

En el trabajo Contribuciones a la instrucción cívica desde la provincia: los libros del profesor Negrete a principios del siglo XX, los autores analizan dos textos de instrucción cívica a inicios del siglo pasado, donde nos dan una muestra de cómo se insertaban los contenidos para la formación del buen ciudadano. A través de estos textos se puede comprender el desarrollo de la sociedad y de los distintos contextos culturales a los cuales iban dirigidos; se mencionan las formas pedagógicas vigentes que se utilizaban para la adecuación de los contenidos por parte de los profesores a los estudiantes. A la par del análisis de los textos del profesor Negrete, también se mencionan algunos datos obtenidos de los expedientes del personaje, que aún se conservan en los archivos de la ciudad de Guadalajara. Sin duda, está presente la visión de los profesores de provincia que aportaron su potencial y compromiso social a la visión que se tenía por los intelectuales de la época, que dirigían las acciones educativas desde el centro del país.

El tercer artículo se denomina Los docentes de primaria y el análisis de fuentes históricas sobre la Conquista de México: habilidades y perspectivas. Es un trabajo de investigación cualitativa que consideró la recuperación de comentarios y experiencias en grupos de discusión de docentes, como intento para conocer las expectativas y el sentido que los profesores dan a las fuentes primarias cuando trabajan contenidos históricos con sus estudiantes. Su propósito es llevar a la reflexión de docentes y estudiantes para perfilar sus 
indagaciones en las fuentes disponibles para conocer de manera directa acontecimientos relacionados con La Conquista desde distintas interpretaciones, haciendo parecer y construir los contenidos históricos con sustento real.

En el trabajo De amores y desamores. Negociación y convivencia en el normalismo rural, I939-I954 el autor nos muestra signos de melancolía ante los recuerdos recuperados de los archivos de las escuelas normales rurales de San Marcos, Zacatecas; Cañada Honda, Aguascalientes, y el Archivo Histórico de la Secretaría de Educación Pública. La correspondencia encontrada y analizada sirve de pretexto para que el autor haga las interpretaciones de los primeros avances de las negociaciones entre estudiantes y autoridades institucionales y gubernamentales. El trabajo está sustentado en fuertes documentales que ya forman parte de la memoria de estas instituciones y en ellas se pueden encontrar varios temas de interés, entre los cuales el autor cita los asuntos relacionados con la salud, la higiene escolar y también los relacionados con el amor, como esa forma de convivencia establecida entre los estudiantes.

La siguiente aportación es Enfoques metodológicos en la investigación histórica: cuantitativa, cualitativa y comparativa, la cual nos lleva a reflexionar en el campo disciplinar de la historia, que responde al complejo de las ciencias comprensivas o del espíritu, de conformidad con la traducción alemana. En la actualidad se ha encontrado que el sentido de la disciplina responde a rescatar al ser -lo humano- como objeto de estudio focal; por lo que se le ha ubicado como una de las ciencias hermenéuticas. En el artículo se 18 realiza un recorrido teórico de las formas o herramientas metodológicas que pueden ser utilizadas para el rescate de lo humano, proponiendo lo cualitativo, lo cuantitativo y el método comparativo. Los enfoques propuestos demuestran que las investigaciones, aun siendo elaborados desde diferentes sustentos teóricos, que responden a diferentes metodologías, pueden y deben de ser utilizados en la investigación de corte histórico. 
La investigación Los heraldos del Centauro. La prensa villista de la ciudad de México (I9I4-I9I5) está basada en fuentes hemerográficas y se realiza con el firme propósito de inmiscuirse en la información que se difundía en la época de la Revolución Mexicana y -en concreto- la relacionada con la aceptación o rechazo que se hacía del Villismo, llegando a concluir que eran escasas las noticias de este caudillo del norte de la República Mexicana. Después de la Convención de Aguascalientes, celebrada en el año de I9I4, la prensa forjó opiniones y aceptación en relación al caudillo -durante los meses de agosto de I9I4 a julio de I9I5- que dejan un espacio temporal de nueve meses y posteriormente la aceptación y la forja de las opiniones de la prensa se direccionaron al apoyo del General Venustiano Carranza, a partir de la pronunciación del Plan de Guadalupe. De nadie es desconocido los avatares y vicisitudes que tuvieron que vencer ambos personajes (Villa y Carranza) y los derroteros que tomaron ambos revolucionarios.

La sección de artículos de investigación cierra con el trabajo Trayectorias, capital social y puntos de inflexión: historia de vida de un adolescente infractor, el cual ofrece los testimonios de una joven infractora, recluida en el Centro de Reinserción Social para Adolescentes Infractores (CERSAI), quien cometió un delito grave de posesión de armas de fuego y de sustancias ilícitas. La narrativa se aboca a conocer los factores o variables que la orillaron a cometer el delito de alto impacto en la sociedad y para ello se requirió establecer un proceso dialógico con entrevistas a profundidad, que nos acercan a conocer el ambiente social y familiar en el cual se desarrolló la vida cotidiana de la hoy interna. El trabajo es sólo una parte de una investigación más amplia que se realiza con la intención de conocer a los adolescentes infractores, para elaborar un programa de actividades educativas que los conduzcan a la integración social y familiar.

Para cerrar el número se incluye la Reseña del Libro Formación docente en Chihuahua, I824-I940 que, como ya es costumbre en la revista Debates por la Historia, se realiza con el fin de dar a conocer los 
avances de este tipo de productos. En el texto podemos encontrar la visión y la historia de la educación en Chihuahua y se recomienda como una lectura amena y bien fundamentada con fuentes históricas primarias. No está por demás mencionar que el texto en mención es resultado de una tesis doctoral que posteriormente fue adaptada al formato de libro, para someterla a concurso en el programa editorial de la Universidad Autónoma de Chihuahua (UACH). La Dra. Esther Soto Pérez es docente e investigadora de la Universidad Pedagógica Nacional del Estado de Chihuahua y en la Facultad de Ingeniería de la UACH. En relación al presentador de la reseña, Dr. Juan Tenorio Urbina, es docente e investigador de la Universidad Pedagógica Nacional del Estado de Chihuahua. Con lo anterior dejamos constancia de la calidad del producto y a la vez invitamos a su lectura.

A manera de cierre de la presente editorial, solo resta mencionar la necesidad de recibir, por parte de los lectores de la revista, sus comentarios y críticas. Igualmente, se les reitera la invitación para publicar sus resultados investigativos en este medio.

\section{Referencias}

Academia, (202I). Verónica Tozzi Thompson. Recuperado de: https://uba.academia.edu/VeronicaTozzi

Betancourt Martínez, F. (2006). Teoría e historia: los signos de una transformación. Observaciones a propósito del diálogo entre historiadores. Estudios de Historia Moderna y Contemporánea de México, (32), I03-I25. Recuperado de: https://www.redalyc.org/articulo.oa?id=94120202004

Blanco Rivero, J. J. (20I2). La historia de los conceptos de Reinhart Koselleck: conceptos fundamentales, Sattelzeit, temporalidad e histórica. Politeia, 35(49), I-33. Recuperado de: https://www.redalyc.org/articulo.oa?id=170029498009 
Metahistorias. (2019). Proyecto de Investigación en Nuevas Filosofías de la Historia. María Inés la Greca. Recuperado de: https://metahistorias.wordpress.com/integrantes/maria-inesla-greca/

UAM-AZC [Universidad Autónoma Metropolitana Azcapotzalco]. (s/f). Departamento de Humanidades UAM-AZC Ficha de personal. Dra. María Norma Durán Rodríguez Arana. Recuperado de: http://humanidades-uama.org.mx/ficha emp.cfm?clave emp=I5

Universidad de Chile (s/f). María Elisa Fernández Navarro. Recuperado de: https:/www.uchile.cl/portafolioacademico/impresion.jsf?username=elisafer

Este artículo se publica bajo una licencia de Creative Commons Reconocimiento-NoComercial 4.0 Internacional, y puede ser usados gratuitamente para fines no comerciales, dando los créditos a los autores y a la revista.

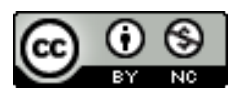

